\title{
Pourquoi promouvoir le développement des régions périphériques et intermédiaires?
}

\author{
Bernard Vachon \\ Université du Québec à Montréal
}

\section{Ce texte constitue un extrait de la communication de Bernard Vachon présentée dans le cadre d'un forum de discussion intitulé «Réussir ensemble le développement de nos collectivités». Ce forum de discussion a eu lieu en février dernier lors de la $19^{\mathrm{e}}$ édition du colloque annuel de la Fondation de l'entrepreneurship : Mentorat 2002 - Générations en action!}

La fracture démographique, économique et sociale entre régions centrales et régions périphériques continue à se creuser. Malgré les efforts concertés de plusieurs milieux et des aides gouvernementales ciblées conduisant à des initiatives innovantes, l'avenir du «Québec des régions» demeure très préoccupant. L'adoption d'une politique globale et intégrée de développement des régions apparaît de plus en plus comme une étape incontournable dans cette lutte aux disparités régionales.

\section{Interrogations de la logique néolibérale}

Face aux difficultés économiques et sociales que traversent les régions périphériques et intermédiaires, la logique marchande de l'idéologie néolibérale s'interroge en ces termes:

- Pourquoi résister au déclin économique et démographique des régions éloignées et intermédiaires?

- Pourquoi ne pas s'accommoder à l'idée de régions désertées, vidées de leurs activités et de leurs populations, abandonnées aux grands espaces sauvages offerts aux citadins comme lieux de récréation et de villégiature?

- Pourquoi ne pas laisser les règles de l'économie organiser seules la mosaïque du peuplement et la distribution des activités de production?

- Pourquoi ne pas aller dans le sens d'une concentration plus poussée de la population et des activités économiques sur les grandes zones métropolitaines du Québec que sont Montréal, Québec, TroisRivières, Hull-Gatineau?

- Pourquoi ne pas souscrire à l'idée que les régions qui survivront seront celles qui adhéreront pleinement aux conditions de la croissance industrielle et urbaine ?

\section{La réponse d'un État responsable}

Parce que les régions (éloignées et intermédiaires), incluant les territoires ruraux, procurent une contribution essentielle à l'organisation territoriale, à la production économique et culturelle et à la qualité de vie de la société globale. Les régions, composées de petites villes, de villages et de vastes espaces naturels :

- assurent une répartition spatiale plus équilibrée de la population et une occupation du territoire en dehors des agglomérations urbaines, évitant ainsi la surcharge des centres;

- élargissent l'éventail des choix de modes de vie, de lieux de travail, de production, de décision et de détente;

- favorisent, par l'activité agricole et d'autres formes d'occupation des sols, l'entretien des paysages et l'accès à l'environnement naturel ;

- assurent un certain niveau d'autosuffisance agroalimentaire ; 
- contribuent à préserver et à transmettre un système de valeurs différent ainsi qu'un patrimoine témoin d'une culture pour laquelle la société demeure attachée ;

- répondent à une volonté profonde d'une proportion importante de la population de vivre dans les collectivités régionales et rurales ;

- constituent des territoires d'accueil pour la nouvelle économie et des segments de population susceptibles de s'établir en dehors des régions centrales;

- représentent une réserve d'éléments et de potentialités pour résoudre demain les crises des grandes agglomérations urbaines.

L'avenir des régions passe essentiellement par l'affirmation vigoureuse de leur utilité économique, sociale, culturelle et écologique. Il passe aussi par le respect de leur spécificité et de leur droit à la juste part des budgets et des aides gouvernementales.

C'est donc dans une véritable reconquête territoriale, démographique, économique et sociale que le développement régional doit s'engager. Pour relever ce défi, les régions doivent miser sur leur volonté et leur capacité d'agir, soit les capacités collectives et individuelles de développement, tout en bénéficiant de politiques nationales de soutien au développement régional.

L'avenir des régions passe essentiellement par l'affirmation vigoureuse de leur utilité économique, sociale, culturelle et écologique. II passe aussi par le respect de leur spécificité et de leur droit à la juste part des budgets et des aides gouvernementales.

\section{Pourquoi une politique de développement des régions?}

L'objectif fondamental à poursuivre: contribuer à la réduction des disparités économiques et sociales entre les régions.

Cette politique se donnera comme axes prioritaires:

1) l'amélioration de la compétitivité des économies régionales dans le but de stimuler la création d'emplois durables;
2) la diversification des économies régionales par des efforts soutenus et des moyens appropriés en faveur d'activités de deuxième et de troisième transformation et le développement de la nouvelle économie incluant le télétravail. Ceci, tout en poursuivant et consolidant les politiques de mise en valeur des ressources naturelles (agriculture, forêt, mines, pêche) qui demeurent des composantes structurelles des économies régionales ;

3) le renforcement de la cohésion sociale et de la situation de l'emploi en particulier par la valorisation des ressources humaines, première richesse des régions, dans le but de contrer l'exode des jeunes et le vieillissement prématuré des populations locales. Un territoire qui n'offre pas d'emplois ne peut retenir sa population.

\section{L'objectif fondamental à poursuivre : contribuer à la réduction des disparités économiques et sociales entre les régions.}

Une politique nationale de développement régional favorisera, à l'échelle de chaque région, la mise en place des conditions préalables aux investissements, c'est-à-dire la réunion des conditions qui confèrent un caractère d'attractivité aux territoires par rapport aux attentes et besoins des investisseurs potentiels, des travailleurs et des familles.

Ceci requiert une connaissance actualisée de la logique de localisation des entrepreneurs, et une bonne compréhension des tendances économiques et sociales de fond qui modifient les rapports de l'activité économique avec l'espace et qui créent de nouvelles opportunités de développement pour les régions, opportunités que les divers programmes et outils contenus dans la politique de développement régional permettront de saisir.

L'État ne crée pas le développement régional, il n'est pas entrepreneur, mais il doit contribuer à créer les conditions propices, c'est-à-dire matérielles, technologiques et humaines, aux investissements privés et ainsi lutter efficacement contre la fracture entre régions centrales et régions périphériques et intermédiaires.

\section{Conditions préalables aux investissements}

Plusieurs conditions doivent être réunies pour conférer à un territoire un caractère d'attractivité à l'égard des investissements. En voici quelques-unes : 
1) un environnement favorable à un esprit d'entreprise ;

2) une population active adaptée aux besoins de la concurrence;

3) des services diversifiés et de qualité offerts aux entreprises et aux personnes (qualité des écoles et des services culturels et de loisirs, services sociaux et de santé, l'ensemble des services de proximité, soutien technique et financier aux entreprises, etc.);

4) des aides en capital ;

5) des infrastructures de transport et de communication adaptées aux besoins, incluant le transport fluvial et les télécommunications. On assurera notamment l'accès à Internet haute vitesse à l'ensemble des villes et villages;

6) les aspects qualitatifs et organisationnels (procédures) du support offert aux entreprises ainsi que l'environnement de la production en général (systèmes productifs locaux);

7) des programmes de qualification de la main-d'œuvre;

8) une qualité de vie assurée par la richesse culturelle et patrimoniale, la mise en valeur de l'environnement bâti et la protection des milieux naturels;
9) l'attention et la place accordées aux jeunes dans les organismes consultatifs et de prise de décision (conseils municipaux, organismes de développement, clubs d'entrepreneurs, etc.).

\section{Une question préalable}

L'adoption d'une politique nationale de développement régional soulève une question préalable d'importance: comme société, souhaitons-nous assurer l'occupation dynamique de toutes les régions du Québec? Sommesnous prêts à consentir les efforts et les ressources nécessaires? Il y a là un choix de société vis-à-vis duquel il faudra prendre position.

La nécessité de procéder à d'importants investissements publics en particulier en matière d'infrastructures, d'équipements et de services pourra requérir certaines modifications des dépenses publiques au profit des régions non centrales. Peut-on envisager promouvoir à cet égard la solidarité territoriale?

Pour répondre à ces questions et à bien d'autres, la tenue d'États généraux sur l'avenir des régions est peut-être une avenue à considérer comme étape préparatoire à l'élaboration de la politique. 